\title{
Mean Field Approximation for Fields of Experts
}

\author{
Muneki YASUDA ${ }^{1, *}$ and Kazuyuki TANAKA ${ }^{2}$ \\ ${ }^{1}$ Graduate School of Science and Engineering, Yamagata University, \\ Yonezawa, Yamagata 992-8510, Japan \\ ${ }^{2}$ Graduate School of Information Sciences, Tohoku University, Sendai 980-8579, Japan
}

Received April 10, 2013; final version accepted June 10, 2013

\begin{abstract}
Fields of experts (FoE) model, which is regarded as a higher-order Markov random field whose clique potentials are modeled by the products of experts, matches spatial structures of natural images well, and therefore, it is an efficient prior of natural images. However, the FoE model does not readily admit efficient inferences because of the complexity of landscape of its energy function. In this paper, we propose an efficient mean field approximation for the FoE model by using a perturbative expansion in statistical mechanics. Our proposed mean field approximation can be applied to the FoE under general settings and can be solved in linear time with respect to the number of pixels. In the latter part of this paper, we apply our method to the image inpainting problem, and we show it gives results being the same or better than ones given by a simple gradient method proposed in the original work.
\end{abstract}

KEYWORDS: probabilistic image processing, image inpainting, fields of experts, mean-field methods, Plefka expansion

\section{Introduction}

Fields of experts (FoE) model, which is regarded as a higher-order Markov random field (MRF) whose clique potentials are modeled by the products of experts [1], is an efficient prior of natural images based on the machine learning [2]. Due to its rich representation power for images, the FoE has been widely adopted by many researchers so far $[3,4]$. The functional forms of the FoEs match spatial structures of natural images well, and therefore, enable us high-quality inferences such as the image denoising, the image inpainting and so on.

Because of complexities of landscapes of energy functions, the FoEs do not readily admit efficient inferences. In order to do efficient inferences with the FoEs, an approximation method based on the belief propagation for the FoE has been developed [5]. However, it has limitations for sizes of cliques because of the computational cost. The computational cost of the belief propagation exponentially increases with increase in the maximum clique size. Therefore, the simple approach of the belief propagation is not practical for a general situation, and then, other approximate approaches have been required. Computing statistical values on FoEs is important for Bayesian treatments with FoEs, for instance, in a denoising with a FoE, we need to use an EM algorithm to estimate hyper-parameters for noise (e.g., noise variance). In the EM algorithm, we need to compute expectations on FoE (i.e., prior) and on a posterior [6]. Thus, demands for an efficient approximation to compute expectations on FoEs has been existed.

In this paper, from statistical mechanical point of view, in order to compute expectations on FoEs we propose a new approximate technique for the FoEs based on a perturbative expansion approach called Plefka expansion [7]. The Plefka expansion is the perturbative expansion technique for MRFs including higher-order MRFs in statistical mechanics. The Plefka expansion can be used to systematically formulate mean field approximations, which are wellknown and important approximate technique, and higher-order approximations for the various range of probabilistic models [8].

We apply the Plefka expansion to the FoEs and propose a mean field approximation for the FoEs in this paper. The proposed mean field approximation has no computational limitations for sizes of cliques unlike the belief propagation mentioned above. Moreover, it can be solved in the linear time with respect to the number of pixels. As an application of our proposal, we attempt image inpainting, which is an image filter reconstructing lost or deteriorated parts of images [9], using our mean field approach. We show that our mean field inpainting gives reconstructions being the same or better than ones obtained by the maximum a posterior (MAP) estimation based on the simple gradient method in the FoE [2] in spite of the fact that our method is the approximation.

The remainder of this paper is organized as follows. In §2, we introduce the FoE model having clique potentials with an arbitrary functional form. This is a generalization of the original FoE model proposed by Roth and Black [2]. In $\$ 3$, 
using the Plefka expansion, we formulate a mean field approximation for the FoE model introduced in the previous section. To apply the Plefka expansion, we define a dual form of the free energy called Gibbs free energy in physics in $\S 3.1$. In the Gibbs free energy, we can treat the FoEs within convex optimization problems. The Plefka expansion is the perturbative expansion of the Gibbs free energy. Using the Gibbs free energy and the Plefka expansion, we derive a mean field approximation for the FoEs in $\S 3.2$. In $\S 4$, we apply our mean field approach to the image inpainting and show numerical results of our mean field reconstructions. Finally, we provide conclusions of this paper and future works in $\S 5$.

\section{Fields of Experts}

Let us consider an image contains $N$ pixels. We label each pixel by $i=1,2, \ldots, N$ on the basis of the raster scan in the image, and we express gray level of pixel $i$ by $x_{i} \in(-\infty, \infty)$. In the image, we define small regions having a certain shape, called cliques. Cliques can generally be defined on all possible regions, having the certain shape, in the image with allowing overlapping among cliques, and they cover the entire image. We label each clique by $c$ and let the set of all cliques be $\mathcal{C}$. Under this setting, the FoE is generally defined as

$$
\begin{aligned}
P_{\mathrm{FoE}}(\boldsymbol{x} \mid \boldsymbol{\Theta}) & :=\frac{1}{Z_{\mathrm{FoE}}(\boldsymbol{\Theta})} \exp (-E(\boldsymbol{x} \mid \boldsymbol{\Theta})), \\
E(\boldsymbol{x} \mid \boldsymbol{\Theta}) & :=-\sum_{c \in \mathcal{C}} \sum_{k=1}^{K} \ln \Phi\left(\boldsymbol{J}_{k}^{\mathrm{t}} \boldsymbol{x}_{c} \mid \boldsymbol{\theta}_{k}\right),
\end{aligned}
$$

where $\boldsymbol{\Theta}=\left\{\boldsymbol{J}_{k}, \boldsymbol{\theta}_{k} \mid k=1,2, \ldots, K\right\}$ is the set of model parameters and $E(\boldsymbol{x} \mid \boldsymbol{\Theta})$ is the energy function of the FoE. The function $\Phi\left(x \mid \boldsymbol{\theta}_{k}\right)$ is a nonnegative potential function with the parameters $\boldsymbol{\theta}_{k}$ and

$$
Z_{\mathrm{FoE}}(\boldsymbol{\Theta}):=\int_{-\infty}^{\infty} \exp (-E(\boldsymbol{x} \mid \boldsymbol{\Theta})) \mathrm{d} \boldsymbol{x}
$$

is the normalization constant. The notation $(\cdots)^{\mathrm{t}}$ denotes the transposition. $\boldsymbol{x}=\left\{x_{i} \mid i=1,2, \ldots, N\right\}$ is the vector of all pixels constructed by the raster scan in the entire image and $\boldsymbol{x}_{c}=\left\{x_{i} \mid i \in c \in \mathcal{C}\right\}$ is the vector of pixels in clique $c$ constructed by the raster scan in the clique. The quantity $K$ represents the number of experts in the FoE and it adjusts the degree of freedom of the model. The parameters $\boldsymbol{J}_{k}=\left(J_{k, 1}, J_{k, 2}, \ldots, J_{k,|c|}\right)^{\mathrm{t}}$, called filters, are the vector having the same dimension as $\boldsymbol{x}_{c}$.

\section{Mean Field Approximation}

In this section, we formulate a mean field approximation for the FoE defined in Eq. (2.1) using a perturbative point of view. Our formulation derived in this section can be generally applied to the FoE without specifying the functional form of the potential function.

\subsection{Gibbs free energy}

By adding a nonnegative constant $\beta$ which is regarded as the temperature in physics, we extend the model (2.1) as

$$
P(\boldsymbol{x} \mid \boldsymbol{\Theta}, \beta):=\frac{1}{Z(\boldsymbol{\Theta}, \beta)} \exp (-\beta E(\boldsymbol{x} \mid \boldsymbol{\Theta})),
$$

where

$$
Z(\boldsymbol{\Theta}, \beta):=\int_{-\infty}^{\infty} \exp (-\beta E(\boldsymbol{x} \mid \boldsymbol{\Theta})) \mathrm{d} \boldsymbol{x}
$$

is the normalization constant. When $\beta=1$, this extended model equal to the original FoE. For the extended model, let us define the free energy,

$$
F(\Theta, \beta):=-\beta^{-1} \ln Z(\Theta, \beta),
$$

which is a cumulant generating function of distribution (3.1). The free energy (3.2) can be introduced the following variational optimization associating with the maximum entropy method.

$$
\beta F(\boldsymbol{\Theta})=\min _{Q} \mathcal{F}[Q] \quad \text { s.t. } \quad \int_{-\infty}^{\infty} Q(\boldsymbol{x}) \mathrm{d} \boldsymbol{x}=1,
$$

where

$$
\mathcal{F}[Q]:=\beta \int_{-\infty}^{\infty} Q(\boldsymbol{x}) E(\boldsymbol{x} \mid \boldsymbol{\Theta}) \mathrm{d} \boldsymbol{x}+\int_{-\infty}^{\infty} Q(\boldsymbol{x}) \ln Q(\boldsymbol{x}) \mathrm{d} \boldsymbol{x}
$$

is known as the variational free energy. 
In order to lead to a dual form of the free energy (3.2), we add new constraints,

$$
\int_{-\infty}^{\infty} x_{i} Q(\boldsymbol{x}) \mathrm{d} \boldsymbol{x}=m_{i} \text { and } \int_{-\infty}^{\infty} x_{i}^{2} Q(\boldsymbol{x}) \mathrm{d} \boldsymbol{x}=v_{i}+m_{i}^{2}
$$

for all $i$, to the variational optimization in Eq. (3.3). This is done by using the Lagrange multiplies expressed as

$$
\begin{aligned}
\mathcal{L}[Q]:= & \mathcal{F}[Q]-\sum_{i=1}^{N} \rho_{i}\left(\int_{-\infty}^{\infty} x_{i} Q(\boldsymbol{x}) \mathrm{d} \boldsymbol{x}-m_{i}\right) \\
& -\sum_{i=1}^{N} \frac{\gamma_{i}}{2}\left(v_{i}+m_{i}^{2}-\int_{-\infty}^{\infty} x_{i}^{2} Q(\boldsymbol{x}) \mathrm{d} \boldsymbol{x}\right)-\lambda\left(\int_{-\infty}^{\infty} Q(\boldsymbol{x}) \mathrm{d} \boldsymbol{x}-1\right),
\end{aligned}
$$

where $\rho, \gamma$, and $\lambda$ are the Lagrange multiplies. The dual form of the free energy (3.2), $G$, is defined by

$$
\begin{gathered}
G(\boldsymbol{m}, \boldsymbol{v}, \boldsymbol{\Theta}, \beta):=\min _{Q} \mathcal{L}[Q]=\max _{\{\boldsymbol{\rho}, \boldsymbol{\gamma}\}}\left\{\boldsymbol{\rho}^{\mathrm{t}} \boldsymbol{m}-\frac{1}{2} \sum_{i=1}^{N} \gamma_{i}\left(v_{i}+m_{i}^{2}\right)\right. \\
\left.-\ln \int_{-\infty}^{\infty} \exp \left(\boldsymbol{\rho}^{\mathrm{t}} \boldsymbol{x}-\frac{1}{2} \sum_{i=1}^{N} \gamma_{i} x_{i}^{2}-\beta E(\boldsymbol{x} \mid \boldsymbol{\Theta})\right) \mathrm{d} \boldsymbol{x}\right\} .
\end{gathered}
$$

Note that $\boldsymbol{m}=\left\{m_{i} \mid i=1,2, \ldots, N\right\}$ and $\boldsymbol{v}=\left\{v_{i} \mid i=1,2, \ldots, N\right\}$ are the vectors constructed by the same manner as $\boldsymbol{x}$, and that $\boldsymbol{m}$ and $\boldsymbol{v}$ are independence variables in this dual form. This dual form is called the Gibbs free energy and it is the convex function with respect to $\boldsymbol{m}$ and $\boldsymbol{v}$. For any $\beta$, the Gibbs free energy has the following important properties: the minimum value of the Gibbs free energy with respect to $\boldsymbol{m}$ and $\boldsymbol{v}$ is equivalent to the free energy, i.e., $\min _{\{\boldsymbol{m}, \boldsymbol{v}\}} G(\boldsymbol{m}, \boldsymbol{v}, \boldsymbol{\Theta}, \beta)=\beta F(\boldsymbol{\Theta}, \beta)$. The values of $m_{i}$ and $v_{i}$ that realize the minimization of the Gibbs free energy are equivalent to the mean and the variance of $x_{i}$ of the distribution (3.1), respectively, i.e., if we denote the values of $\boldsymbol{m}$ and $\boldsymbol{v}$ that minimize the Gibbs free energy by $\boldsymbol{m}^{*}$ and $\boldsymbol{v}^{*}$, respectively,

$$
m_{i}^{*}=\int_{-\infty}^{\infty} x_{i} P(\boldsymbol{x} \mid \boldsymbol{\Theta}, \beta) \mathrm{d} \boldsymbol{x} \quad \text { and } \quad v_{i}^{*}=\int_{-\infty}^{\infty} x_{i}^{2} P(\boldsymbol{x} \mid \boldsymbol{\Theta}, \beta) \mathrm{d} \boldsymbol{x}-m_{i}^{*}
$$

are exactly satisfied for all $i$. Therefore, when $\beta=1, \boldsymbol{m}^{*}$ and $\boldsymbol{v}^{*}$ are equivalent to the exact means and the exact variances of the FoE (2.1), respectively.

\subsection{Plefka expansion}

As mentioned in previous section, the exact mean and the exact variances of the distribution (3.1) can be obtained by minimizing the Gibbs free energy (3.4). However, in general, the minimization of the Gibbs free energy is computationally hard without an approximation. Thus, we expand the Gibbs free energy (3.4) around $\beta=0$, i.e., $G(\boldsymbol{m}, \boldsymbol{v}, \boldsymbol{\Theta}, \beta)=\sum_{n=0}^{\infty} c_{n} \beta^{n} / n$ !, and we approximate it by neglecting higher-order terms. This type of expansion is known as the Plefka expansion in statistical mechanics [7, 10].

The first term of the expansion, $c_{0}$, yields

$$
c_{0}=\sum_{i=1}^{N}\left(\rho_{i}^{*}(0) m_{i}-\frac{\gamma_{i}^{*}(0)}{2}\left(v_{i}+m_{i}^{2}\right)-\frac{\left(\rho_{i}^{*}(0)\right)^{2}}{2 \gamma_{i}^{*}(0)}+\frac{1}{2} \ln \frac{\gamma_{i}^{*}(0)}{2 \pi}\right),
$$

where $\rho^{*}(\beta)$ and $\gamma^{*}(\beta)$ are the values of $\rho$ and $\gamma$ that satisfy the maximum condition in Eq. (3.4), respectively. Therefore, for any $\beta, \rho^{*}(\beta)$, and $\boldsymbol{\gamma}^{*}(\beta)$ satisfy equations

$$
\begin{aligned}
m_{i} & =\int_{-\infty}^{\infty} x_{i} P_{\beta}(\boldsymbol{x} \mid \boldsymbol{m}, \boldsymbol{v}, \boldsymbol{\Theta}) \mathrm{d} \boldsymbol{x} \\
v_{i} & =\int_{-\infty}^{\infty} x_{i}^{2} P_{\beta}(\boldsymbol{x} \mid \boldsymbol{m}, \boldsymbol{v}, \boldsymbol{\Theta}) \mathrm{d} \boldsymbol{x}-m_{i}^{2},
\end{aligned}
$$

for all $i$, where we define

$$
P_{\beta}(\boldsymbol{x} \mid \boldsymbol{m}, \boldsymbol{v}, \boldsymbol{\Theta}) \propto \exp \left(\left(\boldsymbol{\rho}^{*}(\beta)\right)^{\mathrm{t}} \boldsymbol{x}-\frac{1}{2} \sum_{i=1}^{N} \gamma_{i}^{*}(\beta) x_{i}^{2}-\beta E(\boldsymbol{x} \mid \boldsymbol{\Theta})\right) .
$$

When $\beta=0$, from Eqs. (3.6a) and (3.6b), we obtain

$$
\rho_{i}^{*}(0)=m_{i} v_{i}^{-1} \quad \text { and } \quad \gamma_{i}^{*}(0)=v_{i}^{-1} .
$$

From these relations, Eq. (3.5) is reduced to

$$
c_{0}=-\frac{1}{2} \sum_{i=1}^{N}\left(1+\ln 2 \pi v_{i}\right)
$$


The second term of the expansion, $c_{1}$, can be obtained by

$$
\begin{aligned}
c_{1} & =\lim _{\beta \rightarrow 0} \frac{\partial G(\boldsymbol{m}, \boldsymbol{v}, \boldsymbol{\Theta}, \beta)}{\partial \beta} \\
& =-\lim _{\beta \rightarrow 0} \sum_{c \in \mathcal{C}} \sum_{k=1}^{K} \int_{-\infty}^{\infty}\left(\ln \Phi\left(\boldsymbol{J}_{k}^{\mathrm{t}} \boldsymbol{x}_{c} \mid \boldsymbol{\theta}_{k}\right)\right) P_{\beta}(\boldsymbol{x} \mid \boldsymbol{m}, \boldsymbol{v}, \boldsymbol{\Theta}) \mathrm{d} \boldsymbol{x} .
\end{aligned}
$$

When $\beta=0$, with Eq. (3.8), we find

$$
P_{0}(\boldsymbol{x} \mid \boldsymbol{m}, \boldsymbol{v}, \boldsymbol{\Theta})=\prod_{i=1}^{N} \mathcal{N}\left(x_{i} \mid m_{i}, v_{i}\right),
$$

where $\mathcal{N}\left(x \mid \mu, \sigma^{2}\right)$ denotes the one dimensional Gaussian distribution with mean $\mu$ and variance $\sigma^{2}$. By using Eqs. (3.10) and (3.11), we obtain

$$
\begin{aligned}
c_{1} & =-\sum_{c \in \mathcal{C}} \sum_{k=1}^{K} \int_{-\infty}^{\infty} \ln \Phi\left(\boldsymbol{J}_{k}^{\mathrm{t}} \boldsymbol{x}_{c} \mid \boldsymbol{\theta}_{k}\right) \prod_{i \in c} \mathcal{N}\left(x_{i} \mid m_{i}, v_{i}\right) \mathrm{d} \boldsymbol{x}_{c} \\
& =-\sum_{c \in \mathcal{C}} \sum_{k=1}^{K} \int_{-\infty}^{\infty} \mathrm{D} z \ln \Phi\left(\sqrt{\nu_{k, c}} z+\mu_{k, c} \mid \boldsymbol{\theta}_{k}\right),
\end{aligned}
$$

where we define $\int_{-\infty}^{\infty} \mathrm{D} z(\cdots):=\int_{-\infty}^{\infty}(\cdots) \mathcal{N}(z \mid 0,1) \mathrm{d} z$. The expressions $\mu_{k, c}$ and $v_{k, c}$ are defined as

$$
\mu_{k, c}:=\boldsymbol{J}_{k}^{\mathrm{t}} \boldsymbol{m}_{c} \quad \text { and } \quad v_{k, c}:=\left(\boldsymbol{J}_{k}^{(2)}\right)^{\mathrm{t}} \boldsymbol{v}_{c}
$$

for $c \in \mathcal{C}$ and $k=1,2, \ldots, K$, and we define the vector $\boldsymbol{J}_{k}^{(2)}$ by $\boldsymbol{J}_{k}^{(2)}:=\left(J_{k, 1}^{2}, J_{k, 2}^{2}, \ldots, J_{k,|c|}^{2}\right)^{\mathrm{t}}$. The expressions $\boldsymbol{m}_{c}=$ $\left\{m_{i} \mid i \in c \in \mathcal{C}\right\}$ and $\boldsymbol{v}_{c}=\left\{v_{i} \mid i \in c \in \mathcal{C}\right\}$ are the vectors constructed by the same manner as $\boldsymbol{x}_{c}$. In the second line in Eq. (3.12), we use the mathematical property that a linear combination of random variables independently drawn from Gaussian distributions also obeys a Gaussian distribution.

In the context of the Plefka expansion, we can obtain the mean field approximation by neglecting terms higher order than first order and letting $\beta=1$ [7]. Following this sense and using Eqs. (3.9) and (3.12), we obtain the mean field approximation of the FoE (2.1) as

$$
\begin{aligned}
& G(\boldsymbol{m}, \boldsymbol{v}, \boldsymbol{\Theta}, \beta=1) \approx-\frac{1}{2} \sum_{i=1}^{N}\left(1+\ln 2 \pi v_{i}\right) \\
& \quad-\sum_{c \in \mathcal{C}} \sum_{k=1}^{K} \int_{-\infty}^{\infty} \mathrm{D} z \ln \Phi\left(\sqrt{v_{k, c}} z+\mu_{k, c} \mid \boldsymbol{\theta}_{k}\right)=: G_{\mathrm{mf}}(\boldsymbol{m}, \boldsymbol{v}, \boldsymbol{\Theta}) .
\end{aligned}
$$

The values of $\boldsymbol{m}$ and $\boldsymbol{v}$ that minimize the mean field Gibbs free energy, $G_{\mathrm{mf}}(\boldsymbol{m}, \boldsymbol{v}, \boldsymbol{\Theta})$, can be regarded as the mean field approximation of the means and the variances of the FoE (2.1), respectively. The gradients of $G_{\mathrm{mf}}(\boldsymbol{m}, \boldsymbol{v}, \boldsymbol{\Theta})$ with respect to $m_{i}$ and $v_{i}$ are obtained as

$$
\frac{\partial G_{\mathrm{mf}}(\boldsymbol{m}, \boldsymbol{v}, \boldsymbol{\Theta})}{\partial m_{i}}=-\sum_{c \in \mathcal{C}(i)} \sum_{k=1}^{K} J_{k, r_{c}(i)} \int_{-\infty}^{\infty} \mathrm{D} z \frac{\Phi^{(1)}\left(\sqrt{v_{k, c}} z+\mu_{k, c} \mid \boldsymbol{\theta}_{k}\right)}{\Phi\left(\sqrt{v_{k, c}} z+\mu_{k, c} \mid \boldsymbol{\theta}_{k}\right)}
$$

and

$$
\begin{aligned}
& \frac{\partial G_{\mathrm{mf}}(\boldsymbol{m}, \boldsymbol{v}, \boldsymbol{\Theta})}{\partial v_{i}} \\
& \quad=-\frac{1}{2 v_{i}}-\frac{1}{2} \sum_{c \in \mathcal{C}(i)} \sum_{k=1}^{K} J_{k, r_{c}(i)}^{2} \int_{-\infty}^{\infty} \mathrm{D} z \frac{z}{\sqrt{v_{k, c}}} \frac{\Phi^{(1)}\left(\sqrt{v_{k, c}} z+\mu_{k, c} \mid \boldsymbol{\theta}_{k}\right)}{\Phi\left(\sqrt{v_{k, c}} z+\mu_{k, c} \mid \boldsymbol{\theta}_{k}\right)},
\end{aligned}
$$

respectively, where $\mathcal{C}(i)$ is the set of cliques that include pixel $i$, and the notation $r_{c}(i)$ indicates the relative position of pixel $i$ in clique $c$. The notation $\Phi^{(1)}\left(x \mid \boldsymbol{\theta}_{k}\right)$ is defined by $\Phi^{(1)}\left(x \mid \boldsymbol{\theta}_{k}\right):=\partial \Phi\left(x \mid \boldsymbol{\theta}_{k}\right) / \partial x$. The computational cost of minimizing the mean field Gibbs free energy is $O(N)$ if the maximum clique size is negligible as compared with $N$. The one dimensional integrations in Eqs. (3.14) and (3.15) are evaluated by a numerical integration.

\section{Application to Image Inpainting}

The image inpainting is the process of reconstructing lost or deteriorated parts of images [9]. Given a deteriorated image $\boldsymbol{y}$, let the set of lost pixels be $\mathcal{M}$ and suppose that we know which pixels belong to $\mathcal{M}$ in advance. In the Bayesian image inpainting, the likelihood is defined as 


$$
P(\boldsymbol{y} \mid \boldsymbol{x}):=\prod_{i \in \mathcal{M}^{*}} \delta\left(y_{i}-x_{i}\right),
$$

where $\mathcal{M}^{*}$ is the complimentary set of $\mathcal{M}$ and $\delta(x)$ is the Dirac delta function. From the Bayesian theorem, the posterior is

$$
P(\boldsymbol{x} \mid \boldsymbol{y}) \propto P(\boldsymbol{y} \mid \boldsymbol{x}) P_{\mathrm{FoE}}(\boldsymbol{x} \mid \boldsymbol{\Theta})
$$

and the reconstruction based on the MAP estimation is done by maximizing the posterior with respect to $\boldsymbol{x}$ [2].

\subsection{Image inpainting using mean field approximation}

In order to apply the mean field approximation formulated in this paper, instead of the MAP estimation, we introduce a framework of image inpainting similar to a maximum posterior marginals (MPM) estimation, in which the gray levels of pixel $i \in \mathcal{M}, \widehat{x}_{i}$, are estimated by

$$
\widehat{x}_{i}=\int_{-\infty}^{\infty} x_{i} P(\boldsymbol{x} \mid \boldsymbol{y}) \mathrm{d} \boldsymbol{x}=\int_{-\infty}^{\infty} x_{i} P_{\mathrm{FoE}}\left(\boldsymbol{x}_{\mathcal{M}} \mid \boldsymbol{y}_{\overline{\mathcal{M}}}, \boldsymbol{\Theta}\right) \mathrm{d} \boldsymbol{x}_{\mathcal{M}}
$$

where $\boldsymbol{x}_{\mathcal{M}}:=\left\{x_{i} \mid i \in \mathcal{M}\right\}, \boldsymbol{y}_{\overline{\mathcal{M}}}:=\left\{y_{i} \mid i \notin \mathcal{M}\right\}$ and

$$
P_{\mathrm{FoE}}\left(\boldsymbol{x}_{\mathcal{M}} \mid \boldsymbol{y}_{\overline{\mathcal{M}}}, \boldsymbol{\Theta}\right):=\int_{-\infty}^{\infty} P(\boldsymbol{y} \mid \boldsymbol{x}) P_{\mathrm{FoE}}(\boldsymbol{x} \mid \boldsymbol{\Theta}) \mathrm{d} \boldsymbol{x}_{\overline{\mathcal{M}}},
$$

where $\boldsymbol{x}_{\overline{\mathcal{M}}}:=\left\{x_{i} \mid i \notin \mathcal{M}\right\}$. In other words, the estimations are given by the mean values of the posterior (4.1). By using the Gibbs free energy (3.4), the mean values and the variances of the posterior are obtained by

$$
\min _{\{\boldsymbol{m}, \boldsymbol{v}\}} G(\boldsymbol{m}, \boldsymbol{v}, \boldsymbol{\Theta}, \beta=1) \quad \text { s.t. } \quad m_{i}=y_{i}, v_{i}=0 \quad(\forall i \notin \mathcal{M}) .
$$

Employing the mean field Gibbs free energy (3.13) instead of the exact Gibbs free energy in Eq. (4.3), we can construct the algorithm of image inpainting based on the mean field approximation. Our proposed mean field inpainting algorithm is summarized as follows:

1. Given degraded image $\boldsymbol{y}$ having a certain missing region $\mathcal{M}$, initialize $\boldsymbol{m}^{(t)}$ and $\boldsymbol{v}^{(t)}$ with fixing $m_{i}^{(t)}=y_{i}$ and $v_{i}^{(t)}=0$ for all $i \notin \mathcal{M}$ at $t=0$.

2. For all $i \in \mathcal{M}$, update $m_{i}$ and $v_{i}$ by using the gradient decent method with a small positive number $\eta$ :

$$
\begin{gathered}
m_{i}^{(t+1)} \leftarrow m_{i}^{(t)}-\eta \Delta_{m_{i}}^{(t)}, \\
v_{i}^{(t+1)} \leftarrow v_{i}^{(t)}-\eta \Delta_{v_{i}}^{(t)},
\end{gathered}
$$

where $\Delta_{m_{i}}^{(t)}$ and $\Delta_{v_{i}}^{(t)}$ are the gradients calculated by Eqs. (3.14) and (3.15), respectively, at time step $t$. After that, we set $t \leftarrow t+1$.

3. Continue the step 2 until convergence.

The computational cost of this algorithm is $O(|\mathcal{M}|)$ if the maximum clique size is negligible as compared with the size of $\mathcal{M}$.

\subsection{Experiments}

In this section, we demonstrate the mean field image inpainting proposed in the previous section. As the potential function in Eq. (2.1), we employ the student-t type of experts

$$
\Phi\left(x \mid \boldsymbol{\theta}_{k}\right)=\left(1+\frac{x^{2}}{2}\right)^{-\alpha_{k}}, \quad \boldsymbol{\theta}_{k}=\left\{\alpha_{k}\right\},
$$

proposed by Roth and Black in the original FoE [2]. We use the parameters $\left\{\boldsymbol{J}_{k}, \alpha_{k}\right\}$ learned by Roth and Black (their detail values are published in authors' web site). In our experiments in this section, we set $K=8$ and used the $3 \times 3$ square region as the clique. In the following, we compare our results obtained by the mean field approximation with the results obtained by Roth and Black's simple gradient method [2]. Figure 1 shows the results of image inpainting of the scratched image. The results obtained by our mean field reconstruction gives the high quality reconstructed image as much as one reconstructed by Roth and Black's simple gradient method. Figures 2, 3, and 4 show the results of image inpainting. One can see that our method gives the same or better results in spite of the fact that our method is the approximation.

In a sense of the Bayesian estimation, gray levels of missing pixels should be estimated by the MAP estimation of the posterior in Eq. (4.1). However, our mean field method does not search the MAP estimation but the mean values of the pixels of the posterior in the missing region. From our results, in the present model, we can expect that mean values are not so far from MAP solutions. As mentioned before, the Gibbs free energy is the convex function of $\boldsymbol{m}$ and $\boldsymbol{v}$. Although it would not be generally guaranteed that the present mean field approximation completely keeps this convexity, this convex property could be expected to make landscapes of cost functions, which are the mean field Gibbs free energies in the present case, simple and be helpful for searching optimal points. 

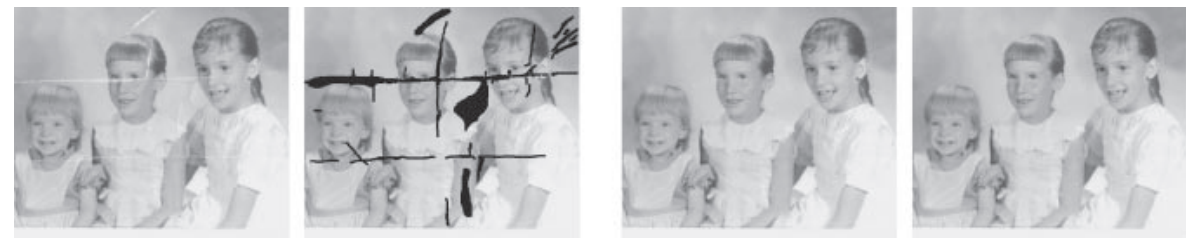

Fig. 1. Results of image inpainting. Original scratched image (left). Masked image by the black regions (second left). Restored image obtained by Roth and Black's simple gradient method (second right). Restored image obtained by our mean field method (right).
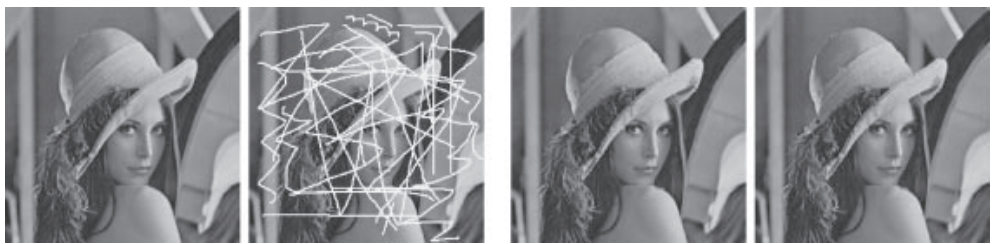

Fig. 2. Results of image inpainting. Original image (left). Degraded image scratched by the white lines (second left). Restored image obtained by Roth and Black's simple gradient method, PSNR = 26.485 [dB] (second right). Restored image obtained by our mean field method, PSNR $=26.650[\mathrm{~dB}]$ (right).
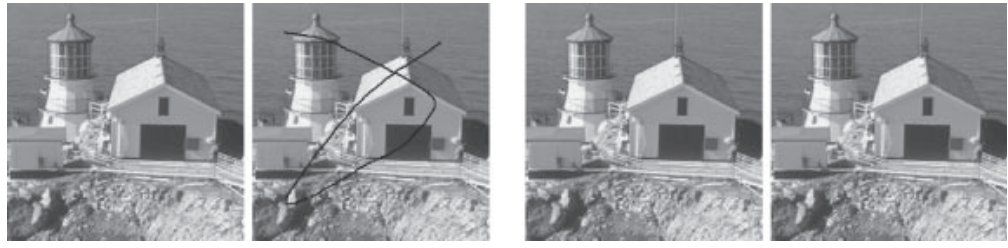

Fig. 3. Results of image inpainting. Original image (left). Degraded image scratched by the black lines (second left). Restored image obtained by Roth and Black's simple gradient method, PSNR $=22.709$ [dB] (second right). Restored image obtained by our mean field method, PSNR = 23.039 [dB] (right).
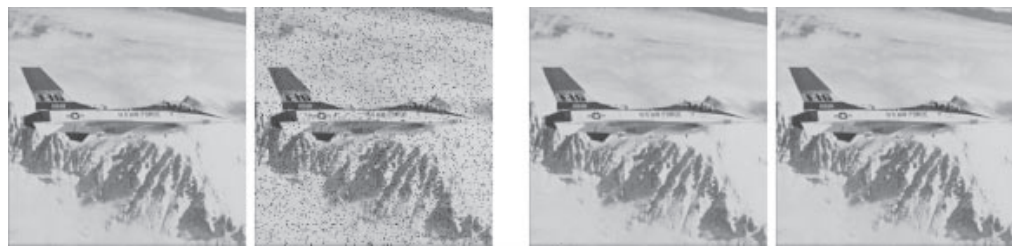

Fig. 4. Results of image inpainting. Original image (left). Degraded image scratched by the black dots (second left). Restored image obtained by Roth and Black's simple gradient method, PSNR $=25.435$ [dB] (second right). Restored image obtained by our mean field method, PSNR $=30.075[\mathrm{~dB}]$ (right).

\section{Conclusions and Future Works}

In this paper, we formulated the Gibb free energy of the FoE described by Eq. (2.1) and, to apply the Plefka expansion, which is the perturbative expansion of the Gibbs free energy, to our Gibbs free energy, we derived the mean field approximation for the FoE. Our mean field approximation can be applied to all FoEs expressed by Eq. (2.1), and it has no limitations for sizes of cliques unlike the belief propagation introduced by the previous work [5]. Therefore, our mean field method is expected to be more practical and to have various applications. Moreover, we applied our mean field method to the image inpainting. Our mean field image inpainting gave the same or better results than Roth and Black's gradient method. Since our mean field method can compute expectations on FoEs, we can immediately apply it to Bayesian treatments with FoEs to estimate hyper-parameters, for example, EM algorithms. This should be addressed in the next study.

The present naive mean-field level approximation is interpreted as a simple Gaussian approximation in which constraints for higher-order moments (higher than mean values and variances) are neglected. Thus, the use of presented formulation might be limited to some simple models. However, the present Plefka expansion scheme enables us to systematically obtain higher-order coefficients and to formulate a higher-order approximation of the FoE. In the sense 
of the Plefka expansion, the neglected effects are in higher-order coefficients and the higher-order approximation is expected to give better results. Hence, we are interested in the higher-order coefficients of the present Plefka expansion, especially the second-order coefficient which is known as Thouless-Anderson-Palmer (TAP) approximation in statistical physics [7, 11]. The present framework of the Gibbs free energy and the Plefka expansion can be applied to not only the inference but also the machine learning. Because, given a training data set, the free energy (3.2) appears in the log-likelihood function which is the cost function in the maximum likelihood estimation. Constructing learning algorithms for FoEs based on the present framework is addressed in the future.

We employed the gradient decent method to minimize the mean field Gibbs free energy in Eq. (3.13). It is known that the gradient decent method has a slow convergence. As a result, our algorithm is not very fast at the present stage. Thus, it is important to investigate other strategies of minimizing the mean field Gibbs free energy, for instance, another gradient method such as the conjugate gradient method or a message-passing method like belief propagations.

\section{Acknowledgments}

This work was partly supported by Grants-in-Aid (No. 24700220 and No. 22300078) for Scientific Research from the Ministry of Education, Culture, Sports, Science and Technology, Japan.

\section{REFERENCES}

[1] Welling, M., Hinton, G., and Osindero, S., "Learning sparse topographic representations with products of student-t distributions," Adv. Neural Inf. Process. Syst., 15: 1359-1366 (2003).

[2] Roth, S., and Black, M. J., "Fields of experts," Int. J. Comput. Vision, 82: 205-229 (2009).

[3] Weiss, Y., and Freeman, W. T., "What makes a good model of natural images," Proc. IEEE Conf. Computer Vision and Pattern Recognition (CVPR) (2007).

[4] Samuel, K. G. G., and Tappen, M., "Learning optimized map estimates in continuously-valued MRF models," Proc. IEEE Comput. Soc. Conf. Computer Vision and Pattern Recognition (CVPR) (2009).

[5] Lan, X., Roth, S., Huttenlocher, D. P., and Black, M. J., "Efficient belief propagation with learned higher-order markov random fields," Proc. ECCV2006, 2: 269-282 (2006).

[6] Tanaka, K., "Statistical-mechanical approach to image processing," J. Phys. A: Math. Gen., 35: R81-R150 (2002).

[7] Plefka, T., "Convergence condition of the tap equation for the infinite-ranged Ising spin glass model," J. Phys. A: Math. Gen., 15: 1971-1978 (1982).

[8] Yasuda, M., and Tanaka, K., “TAP equation for non-negative Boltzmann machine,” Philos. Mag., 92: 192-209 (2012).

[9] Bertalmío, M., Sapiro, G., Caselles, V., and Ballester, C., "Image inpainting," Proc. SIGGRAPH2000 (2000).

[10] Georges, A., and Yedidia, J. S., "How to expand around mean-field theory using high-temperature expansions," J. Phys. A: Math. Gen., 24: 2173-2192 (1991).

[11] Thouless, D. J., Anderson, P. W., and Palmer, R. G., "Solution of 'solvable model of a spin glass'," Philos. Mag. 35: 593-601 (1977). 